
\title{
25 Research Soure \\ Comparison of general maternal and neonatal conditions and clinical outcomes between embryo transfer and natural conception
}

Haiyan Pan

Guangdong Medical University

\section{Xingshan Zhang}

Guangdong Medical University

Jiawei Rao

Guangdong Medical University

Bing Lin

Shunde Women and Children's Hospital of Guangdong Medical University

JieYun He

Shunde Women and Children's Hospital of Guangdong Medical University

\section{Xingjie Wang}

Guangdong Medical University

\section{Fengqiong Han}

Shunde Women and Children's Hospital of Guangdong Medical University

Jinfeng Zhang ( $\nabla$ jinfeng_zhangjf@163.com )

Shunde Women and Children's Hospital of Guangdong Medical University https://orcid.org/0000-00019142-4148

\section{Research}

Keywords: embryo transfer, natural conception, clinical outcome, assisted reproductive technology

Posted Date: January 8th, 2020

DOl: https://doi.org/10.21203/rs.2.20397/v1

License: (c) (i) This work is licensed under a Creative Commons Attribution 4.0 International License. Read Full License

Version of Record: A version of this preprint was published at BMC Pregnancy and Childbirth on July 27th, 2020. See the published version at https://doi.org/10.1186/s12884-020-03066-9. 


\section{Abstract}

Objective: To examine the differences between pregnant women who underwent embryo transfer (ET) and those who conceived naturally, as well as differences in their respective babies, and to determine the causes for these differences, to provide recommendations for women who are planning to undergo ET.

Methods: A retrospective cohort study was performed of women who had received ET and those who had natural conception (NC) who received medical services during pregnancy and had their babies delivered at the Shunde Women and Children's Hospital of Guangdong Medical University, China between January 2016 and December 2018. Each case in an ET group of 321 women was randomly matched with three cases of NC (963 cases) who delivered on the same day. The demographic information, past history, pregnancy and delivery history, and maternal and neonatal outcomes of the two groups were compared using univariate analysis.

Results: Age, occupation, duration of hospitalization, number of pregnancies, number of miscarriages, induced abortion, ectopic pregnancy, gestational diabetes mellitus, preeclampsia, gestational anemia, pregnancy risk, mode of fetal delivery, and number of births were significantly different between the two groups (all $P<0.05$ ). However, there were no significant differences in the disease, allergy and blood transfusion histories of the pregnant women, or differences in prevalence of gestational hypothyroidism, gestational respiratory infection, premature rupture of membrane, placental abruption, fetal death, stillbirth, amniotic fluid volume and amniotic fluid clarity between the two groups (all $P>0.05$ ). The percentages for low birth weight and premature birth were significantly higher in the ET group than in the NC group, while the incidence of nuchal cord was higher in the NC group (all $P>0.05$ ). In contrast, infant gender and prevalence of fetal macrosomia, fetal anomaly, neonatal asphyxia, and extremely low birth weight were not significantly different between the two groups (all $P>0.05$ ).

Conclusions: The clinical outcomes of mothers and the birth status of infants were better in the NC group than in the ET group. Maternal health must be closely monitored and improved in the ET group to reduce the incidence of gestational comorbidity and enhance the quality of fetal life.

\section{Introduction}

Increasingly, couples are turning to assisted reproductive technology (ART) for help with conceiving and ultimately giving birth to a healthy live baby of their own ${ }^{[1]}$. In recent years, there has been increasing concern regarding the safety of ART, due to the potential health impact on these infants. At present, multiple studies have suggested that in vitro fertilization (IVF) pregnancies may be at increased risk for preterm birth, low birth weight, congenital anomalies, perinatal mortality and several other pregnancyrelated complications compared to unassisted pregnancies ${ }^{[2]}$.Concerns have been raised over an increased risk of adverse maternal outcomes like gestational diabetes mellitus and preeclampsia in ART populations as compared with the natural conception group ${ }^{[3]}$. 
In this study, we conducted a questionnaire survey of eligible subjects to determine the differences in clinical outcomes of mothers and their newborns between embryo transfer (ET) and natural conception (NC) and to provide data information for the embryo transfer information database of Shunde and reference information for the mother who is about to undergo embryo transfer.

\section{Methods}

\section{Subjects}

The subjects for this study were 321 women who had ET and their babies and 963 women who conceived naturally (natural conception, NC) and their babies, who delivered in the Shunde Women and Children's Hospital of Guangdong Medical University between January 2016 and December 2018.

\section{Method}

The general information of the 321 women who underwent ET in the hospital and their babies was subjected to a retrospective analysis. Each ET case was randomly matched with three NC cases born on the same day as the ET case, and a total of $963 \mathrm{NC}$ cases were used as controls. Simple random sampling was used. This proportion could achieve satisfactory research results and the workload was more appropriate. The general conditions and clinical outcomes of the women during their pregnancy and their babies were compared between the ET and NC groups.

\section{Source of information}

Original data from the admission records were transferred to a paper data collection questionnaire form. The same information was retrieved for both groups and included general demographic information, past medical history, history of pregnancy and delivery, and clinical outcomes of the mothers (maternal comorbidities and current delivery records) and infants (gender, premature birth, birth weight, birth defects, and neonatal asphyxia).

\section{Statistical analysis}

Data were entered using Excel and EpiData 3.1, and were statistically analyzed using SPSS 22.0 (IBM). Categorical data were expressed as frequency (\%) and compared using the $\boldsymbol{c}^{2}$ test. Continuous data were expressed as mean \pm standard deviation $( \pm s)$ and normally distributed data were compared using the independent samples $t$-test. $P<0.05$ was considered statistically significant.

\section{Results}

\section{Demographic information}

Age, occupation, marital status and educational background were significantly different between the two groups of women $(P<0.05)$. The percentage of women who were $\geq 35$ years old while pregnant was 
higher in the ET group. However, the number of unmarried women and the percentage of women with secondary school education were higher in the NC group (See Table 1, Additional File).

\section{Comparison of medical past history between the embryo transfer and natural conception groups}

There were no significant differences in the history of disease, allergy and blood transfusion between the two groups ( $P>0.05)$. However, the proportion of women with a history of infection or surgery was significantly higher in the ET group $(P<0.05)$ (See Table 2, Additional File).

\section{Comparison of pregnancy and delivery history between the embryo transfer and natural conception groups}

The number of pregnancies, miscarriages, spontaneous abortions, induced abortions and ectopic pregnancies was significantly different between the ET and NC groups (all $P<0.05$ ). The percentage of women who had experienced $>1$ pregnancy or $\geq 1$ miscarriage was higher in the NC group. In contrast, spontaneous abortion and ectopic pregnancy had occurred more in the ET group. There were no significant differences between the two groups in the number of fetal deaths and stillbirths $(P>0.05$; See Table 3, Additional File).

\section{Comparison of maternal clinical outcomes between the embryo transfer and natural conception groups}

\section{Maternal comorbidities}

The incidence rates of gestational diabetes mellitus (GDM), gestational anemia and preeclampsia were significantly higher in the ET group than in the NC group (all $P<0.05$ ). There were no significant differences in the incidence of other maternal comorbidities between the two groups $(P>0.05)$. The incidence of threatened labor was significantly higher in the NC group $(P<0.05$; See Table 4, Additional File).

The number of days from admission to birth for treatment to prevent miscarriage was significantly higher in the ET group than in the NC group ( $P<0.05$; See Table 5, Additional File).

\section{Delivery records}

The number of gestational weeks, pregnancy risk classification, mode of delivery, and number of births were significantly different between the ET and NC groups (all $P<0.05$ ). Compared with the NC group, the ET group had higher percentages of delivery at $<37$ weeks, class III risk, cesarean section and twin birth (39.6\%). The NC group had comparable proportion of all three classes of risks, a higher proportion of vaginal deliveries, and a higher proportion of single births (98.2\%). There were no significant differences in the volume and clarity of amniotic fluid between the two groups ( $P>0.05$; See Table 6, Additional File).

There was no significant difference between the ET and NC groups in the causes of cesarean section ( $P>0.05$; See Table 7, Additional File). 


\section{Comparison of neonatal clinical outcomes between embryo transfer and natural conception groups}

The percentages for low birth weight and premature birth were significantly higher in the ET group than in the NC group, while the incidence of nuchal cord was higher in the NC group (all $P<0.05$ ). In contrast, there were no significant differences in percentages between the two groups for baby gender, fetal macrosomia, fetal anomaly, neonatal asphyxia and extremely low birth weight (all $P>0.05$; See Table 8, Additional File).

\section{Discussion}

A total of 22775 babies were born between January 2016 and December 2018 in the Shunde Women and Children's Hospital of Guangdong Medical University. The proportion of infants conceived by embryo transfer was $1.4 \%$. In this study, we found that the proportion of geriatric pregnancies was higher in the ET group than in the NC group, as well as the overall age of pregnant women (33.23 \pm 4.59 vs $30.16 \pm$ 5.19 years). Geriatric IVF pregnancy was previously reported as having poorer outcomes than IVF pregnancy in younger women. One study found that among women of childbearing age, those aged 2030 years had the best IVF outcomes and women aged 40 years and older had poor IVF outcomes and a higher rate of miscarriage ${ }^{[4]}$. Secondary school includes junior high school and senior high school. China has a nine-year compulsory education system, and all children can go to junior high school for free, so the proportion of women with secondary school education is the highest. As women age, a decline in oocyte production and quality (known as ovarian aging) becomes the primary cause of poorer IVF outcome ${ }^{[5]}$. We observed that the percentage of women with one pregnancy only was higher in the ET group (48.0\%) than in the NC group (31.0\%), which was consistent with the findings of Egbe et al. ${ }^{[6]}$ This difference may be attributable to the various causes of infertility in the ET group, meaning women in the ET group were more likely to be having their first pregnancy than those in the NC group. Ectopic pregnancy is the primary cause of early maternal morbidity and mortality, accounting for $1-2 \%$ of all pregnancies and ectopic pregnancy incidence has drastically increased with the advancement of ART [7]. The fallopian tube is the most common site of ectopic implantation ${ }^{[8]}$, and about $1.5-2.1 \%$ of patients with ectopic pregnancy have undergone IVF ${ }^{[7]}$. Our findings showed that the percentage of women with a history of ectopic pregnancy was higher in the ET group (15.3\%) than in the NC group (2.7\%), which may be associated with previous ectopic pregnancy, history of infertility, history of surgery or the use of intrauterine contraceptive device ${ }^{[9]}$.

For gestational comorbidity, the study by Kouhkan et al. ${ }^{[10]}$ showed that women who underwent ET needed more insulin than those in the NC group. Insulin resistance and glucose intolerance during pregnancy can lead to the development of GDM, which explains the higher incidence of GDM in the ET group $(42.1 \%)$ relative to the NC group $(26.4 \%)$ in our study.

Preeclampsia is a pregnancy-specific disease with a global prevalence of $5-8 \%$. It is one of the leading causes of maternal and perinatal morbidity and mortality worldwide, causing 50,000 to 60,000 deaths 
every year ${ }^{[11]}$. In our study, preeclampsia incidence was higher in the ET group. Preeclampsia is a multisystemic syndrome, and its pathogenesis and pathophysiology involve both genetic and environmental factors ${ }^{[12]}$. If preeclampsia is not effectively treated in a timely manner, it may endanger the life of the mother and infant or cause sequelae in the short term, and subsequently affect the health of the mother in the long term. Therefore, pregnant women are recommended to complete all prenatal examinations and adhere to a healthy routine and lifestyle. We also found that the incidence of gestational anemia and the number of days of treatment to prevent miscarriage were higher in the ET group ( $26.0 \%$ and $8.45 \pm 7.408$, respectively) than in the NC group ( $4.1 \%$ and $4.80 \pm 4.026$, respectively). Most pregnant women who required treatment for miscarriage prevention had threatened labor. The pathogenesis of gestational anemia has been shown to be associated with the age and educational background of pregnant women, as well as a history of ectopic pregnancy ${ }^{[13]}$. This was consistent with our results, which found that age and incidence of ectopic pregnancy were both higher in the ET group than in the NC group. In addition, ET itself may be a factor associated with gestational anemia. A previous study has demonstrated that severe anemia in pregnant women can lead to premature labor, spontaneous abortion, low birth weight and fetal death ${ }^{[14]}$. Therefore, implementation of measures to prevent anemia is recommended for women who plan to undergo ET, to ensure maternal and neonatal health.

Analysis of the delivery records revealed that the proportion of women with a high-risk (class III) pregnancy was significantly higher in the ET group (81.3\%) than in the NC group (44.9\%). Pregnancy risk is primarily classified based on the general conditions of the pregnant woman (age, history of miscarriage, and history of adverse pregnancy), gestational comorbidities (hypertension, anemia, and respiratory infection), and gestational complications (threatened premature labor, GDM, and fetal macrosomia). Age, ART, and twin pregnancy may be the causes for the higher pregnancy risk in the ET group compared with the NC group. The dominant mode of delivery was cesarean section in the ET group (78.2\%) and vaginal delivery in the NC group (81.0\%). The rate of twin birth was higher in the ET group $(39.6 \%)$ than in the NC group (1.8\%); twin birth is known to be associated with ET. Twin pregnancy imposes certain risks to both maternal and neonatal health, so this issue needs to be carefully considered in the application of ET.

A study by Zhu et al. ${ }^{[15]}$ demonstrated that embryo transfer was associated with higher incidence of premature birth, low birth weight and small-for-gestational age infants. Here, we found that premature birth and low birth weight were observed in $38.8 \%$ and $36.5 \%$ of women with ET, respectively. The risk of premature birth is higher among women undergoing IVF, but such risk is mostly believed to be a secondary consequence of the significant increase in multiple pregnancies ${ }^{[16]}$. Our study also showed that the ET group had a higher incidence of twin pregnancy. Furthermore, Qin et al. ${ }^{[17]}$ demonstrated that low birth weight was positively correlated with ART, which was consistent with our findings that the proportions of premature and low birth weight babies were higher in the ET group (24.9\% and $18.7 \%$, respectively) than in the NC group ( $14.1 \%$ and $11.2 \%$, respectively) when we compared neonatal clinical outcomes. Compared with the ET group, the NC group had a higher percentage of umbilical cord 
wrapping around the baby's neck and foot but a lower percentage of umbilical cord torsion. The umbilical cord is a conduit between the fetus and the placenta that mediates substance exchange between the fetus and mother. Umbilical cord complications are generally considered to be the root cause for chronic intrauterine hypoxia, reduced fetal movement, growth retardation and oligohydramnios ${ }^{[18]}$. Therefore, regular prenatal examinations are recommended for pregnant women, especially ultrasound examination during the second and third trimesters of pregnancy, to ensure early identification of umbilical cord abnormality.

In summary, the ET group had poorer maternal clinical outcomes than the NC group and higher incidences of premature birth and low birth weight. These differences may be associated with maternal physical fitness, nutritional status, financial status, past health status, ET, and higher rates of reported adverse outcomes due to closer monitoring of pregnant women with ET. Women who plan to have ET are recommended to undergo the procedure at an appropriate reproductive age, maintain physical fitness and good nutrition, and take good prenatal care.

\section{Conclusions}

The clinical outcomes of mothers and the birth status of infants were better in the NC group than in the ET group. Maternal health must be closely monitored and improved in the ET group to reduce the incidence of gestational comorbidity and enhance the quality of fetal life.

\section{Declarations}

\section{Ethics approval and consent to participate}

All procedures performed in studies involving human participants were in accordance with the ethical standards of the institutional and/or national research committee and with the 1964 Helsinki declaration and its later amendments or comparable ethical standards.

\section{Consent for publication}

Not applicable

\section{Availability of data and materials}

The datasets used and/or analyzed during the current study are available from the corresponding author on reasonable request.

\section{Competing interests}

The authors declared that they have no conflicts of interest to this work.

\section{Funding}


Medical Science and Technology of Guangdong Province (B2019087), Young Innovative Talents Project of Guangdong Province (2018KQNCX096), and Nature Science Foundation of Guangdong Province (2019A1515010875).

\section{Authors' contributions}

HYP participated in the design of this study and wrote the manuscript. JFZ and FQH carried out the study and collected important background information. XSZ, JWR and XJW collected the data. BL and JYH processed the data. All authors read and approved the final manuscript.

\section{Acknowledgements}

To all the patients who selflessly contributed to the conduct of the study and especially to HYP, JFZ and $\mathrm{FQH}$, for contributing with their experience and enthusiasm.

\section{Authors' information}

HYP, Associate Professor, Master Tutor, School of Public Health, Guangdong Medical University. JFZ, FQH, BL and JYH, hospital staff, Shunde Women and Children's Hospital of Guangdong Medical University. XSZ, JWR and XJW, Graduate Student of Guangdong Medical University.

\section{Abbreviations}

ET: embryo transfer

NC: natural conception

IVF: in vitro fertilization

ART: assisted reproductive technology

IBM: International Business Machines Corporation

GDM: gestational diabetes mellitus

\section{References}

1. Farquhar C, Marjoribanks J. Assisted reproductive technology: an overview of Cochrane Reviews. Cochrane Database Syst Rev. 2018; 8(8).

2. Kondapalli LA, Perales-Puchalt A. Low birth weight: is it related to assisted reproductive technology or underlying infertility?. Fertil Steril. 2013;99(2):303-310.

3. Jie Z, Yiling D, Ling Y. Association of assisted reproductive technology with adverse pregnancy outcomes. Iran J Reprod Med. 2015,13(3):169-180. 
4. Gleicher N, Kushnir VA, Albertini DF, Barad DH. Improvements in IVF in women of advanced age. J Endocrin. 2016; 230 (1): 1-6.

5. Thomas Obinchemti Egbe, Guy Sandjon, Clovis Ourtchingh,et al. In-vitro fertilization and spontaneous pregnancies: matching outcomes in Douala, Cameroon [J].Fertility Research and Practice,2016,2(1):1-8.

6. Nicole Yoder,Reshef Tal,Ryan Martin.Abdominal ectopic pregnancy after in vitro fertilization and single embryo transfer: a case report and systematic review[J].Reproductive Biology and Endocrinology, 2016, 14, 69.

7. Danielle M.Panelli,Catherine H.Phillips,Paula C.Brady,et al. Incidence, diagnosis and management of tubal and nontubal ectopic pregnancies: a review[J].Fertility Research and Practice,2015,1,15.

8. Cheng Li,Wei-Hong Zhao,Qian Zhu,et al. Risk factors for ectopic pregnancy: a multi-center casecontrol study[J].BMC Pregnancy And Childbirth, 2015, 15: 187.

9. Azam Kouhkan,Mohammad E.Khamseh,Reihaneh Pirjani,et al. Obstetric and perinatal outcomes of singleton pregnancies conceived via assisted reproductive technology complicated by gestational diabetes mellitus: a prospective cohort study[J].BMC Pregnancy and Childbirth, 2018, 18 (1): 495506.

10. P Gathiram and J Moodley.Pre-eclampsia:its pathogenesis and pathophysiology [J].Cardiovascular Journal of Africa , 2016, 27(2): 71-78.

11. Roberto Romero and Tinnakorn Chaiworapongsa.Preeclampsia:a link between trophoblast dysregulation and an antiangiogenic state[J].Journal of Clinical Investigation,2013,123(7):27752777.

12. Wenjing Hao. Investigation and analysis of the pathogenic factors and perinatal outcomes of gestational anemia in China [D]. Capital Medical University, 2016, 1-48.

13. SIFAKIS and G.PHARMAKIDES.Anemia in pregnancy [J].Annals of the New York Academy of Sciences,2000,900(1):125-136.

14. Linling Zhu,Yu Zhang, Yifeng Liu,et al. Maternal and Live-birth Outcomes of Pregnancies following Assisted Reproductive Technology: A Retrospective Cohort Study[J].Scientific Reports, 2016, 6: 35141.

15. Adam J.Fechner,Kelecia R. Brown,Ndidiamaka Onwubalili,et al. Effect of single embryo transfer on the risk of preterm birth associated with in vitro fertilization[J].Journal of Assisted Reproduction Genetics, 2015, 32 (2): 221-224.

16. Jiabi Qin,Hua Wang,Hua Wang,et al. Pregnancy-related complications and adverse pregnancy outcomes in multiple pregnancies resulting from assisted reproductive technology: a meta-analysis of cohort studies[J].Fertility And Sterility, 2015, 103 (6): 1492-1508.

17. C.Fleisch1 and Thomas Hoehn.Intrauterine fetal death after multiple umbilical cord torsioncomplication of a twin pregnancy following assisted reproduction [J].Journal of Assisted Reproduction Genetics, 2008, 25 (6): 277-279. 


\section{Supplementary Files}

This is a list of supplementary files associated with this preprint. Click to download.

- AdditionalFile.xlsx 